\title{
Revista de Oncología: una nueva etapa
}

La Revista de Oncología, portavoz de la Federación de Sociedades Españolas de Oncología, se ha hecho merecedora en sus 2 años de existencia de un lugar destacado en las publicaciones dedicadas a la Oncología. El dinamismo, la dedicación y el aporte de iniciativas por parte del doctor Rafael Rosell, director; del doctor Juan Carlos Lacal, secretario general, y de los redactores jefe de las distintas especialidades han conseguido un magnífico logro. Dicho logro es el disponer cada 2 meses de un nuevo ejemplar de una revista con prestigio, consolidada, ampliamente leída y consultada por los profesionales de la Oncología y disciplinas afines.

Se están haciendo todos los esfuerzos posibles para seguir incrementando la calidad científica de los originales publicados. Creo sinceramente que se está consiguiendo y que nuestro país tiene un potencial investigador alto, aunque no siempre suficientemente reconocido. Pero la calidad y el rigor científico deben ir de la mano de un diseño atractivo y una difusión amplia. Para ello se ha estimulado la publicación indistinta de artículos en español y en inglés que facilite la lectura más allá de nuestras fronteras.

Pero el ejemplar que hoy llega a vuestras manos tiene un significado muy especial. Es por primera vez el fruto de la colaboración estrecha entre países hermanos. El acuerdo entre FESEO y el Instituto Mexicano de Cancerología, suscrito en La Coruña durante el
IV Congreso Nacional de FESEO el pasado mes de octubre, ha propiciado la fusión de las revistas de cada organismo en una nueva REVISTA DE ONCOLOGÍA que será el portavoz de FESEO y del Instituto Nacional de Cancerología de México. La edición será simultánea en los 2 países y el contenido único.

Con este acuerdo, que nos llena de satisfacción, creemos que se da un gran paso adelante en la difusión del conocimiento de las ciencias oncológicas, tanto en su vertiente clínica como básica, en el amplio ámbito de la comunidad hispanoamericana. Al mismo tiempo, la publicación de artículos en inglés, lengua común del mundo científico, también facilitará la difusión en el mundo no hispanohablante.

Mi agradecimiento personal e institucional a todos los que han hecho posible el inicio de esta nueva etapa, tanto a los equipos de las revistas como a DOYMA, que ha puesto los medios personales y materiales para este proyecto.

Se inicia una nueva e ilusionante andadura. Para que sea realidad falta lo más importante: que todos confiemos en este proyecto y enviemos nuestros mejores trabajos científicos a la Revista de Oncología.

Un cordial saludo. 